\title{
Physicochemical and Sensory Properties of Nanopowdered Chitosan- Added Maribo Cheese during Ripening
}

\author{
Hee-Yeon Kim, Yu-Tae Jeong ${ }^{1}$, In-Hue Bae ${ }^{1}$, and Hae-Soo Kwak* \\ Department of Food Science and Technology, Sejong University, Seoul 143-747, Korea \\ ${ }^{1}$ Department of Animal Science and Technology, Sunchon National University, Jeonnam 540-742, Korea
}

\begin{abstract}
Nanopowdered chitosan (NPC) has high biological activities, such as blood cholesterol lowering effect and antidiabetic activity. This study is carried out to determine the effects of nano-powdered chitosan-added Maribo cheese (NCMC) for the physicochemical properties and sensory analysis during its ripening at $14^{\circ} \mathrm{C}$ for $6 \mathrm{mon}$. From the results, the moisture and fat levels are not significantly influenced from the addition of chitosan $(p>0.05)$, but ash contents increased with increasing chitosan concentrations and the protein contents decreased with increasing chitosan concentrations. In the short-chain fatty acids analysis during the ripening, the total production is initially $13.79 \mathrm{ppm}$ in $0.2 \% \mathrm{NCMC}$ and $13.81 \mathrm{ppm}$ in control, and their levels have steadily increased to 59.94 and $53.11 \mathrm{ppm}$, respectively. For the color levels, the L* values decreased, while the $\mathrm{a}^{*}$ and $\mathrm{b}^{*}$ values significantly increased during ripening for all samples $(p<0.05)$. In texture analysis, the hardness and gumminess of NCMC significantly decreased as compared to the control during ripening $(p<0.05)$, while the cohesiveness, springiness and chewiness were not significantly different among the treatments $(p>0.05)$. In sensory analysis, the butyric off-flavor and bitterness increased slightly with increasing concentrations of NCMC during ripening. The overall acceptability of $0.2 \% \mathrm{NCMC}$ held the highest score amongst the samples during the ripening. From the results obtained, the $0.2 \% \mathrm{NCMC}$ was preferred during the ripening and observed the possibility of functional cheese.
\end{abstract}

Key words: maribo cheese, nanopowdered chitosan, ripening

\section{Introduction}

Chitosan, the main derivatives of chitin, is a linear aminopolysaccharide composed primarily of repeating unit of $\beta$-(14)-linked-N-acetylglucosamine. Various functions of chitosan, such as cholesterol lowering (Gallaher et al. 2000), antitumor (Qin et al. 2002), immuno-enhancing, antidiabetic (Hayashi and Ito, 2001), antihypertensive, wound healing, antifungal, and antimicrobial activity, have been reported in numerous studies. However, when chitosan is applied in the food industry, there has been difficulty in dissolving it in water. To increase solubility of chitosan, it has to be modified by chemical techniques. However, chemical modification can cause losing original physicochemical and biochemical properties (Sugimoto et al. 1998). Therefore, nanotechnology may be one of the solutions for this problem.

\footnotetext{
*Corresponding author: Hae-Soo Kwak, Department of Food Science and Technology, Sejong University, Seoul 143-747, Korea. Tel: 82-2-3408-3226, Fax: 82-2-3408-4319, E-mail: kwakhs@ sejong.ac.kr
}

Nano-sized substances could increase solubility and bioavailability due to the extremely increased surface area. (Chaudhry et al. 2008, Park et al. 2007). To find the effect of hypoglycemic, nanopowdered chitosan $(562 \mathrm{~nm})$ was studied in which 2\% NPC-treated group had 79\% higher secretion in insulin than that of commercial powdered chitosan (Seo et al., 2010). In addition, chitosan nanopowder had better hypolipidemic effect than ordinary chitosan in rats (Zhang et al., 2013). Total cholesterol lowering effect from nanochitosan in rat was conducted in which NPC was reduced by $46.6 \%$ and PC by $18.6 \%$ (Park et al., 2010). NPC as a functional ingredient was applied in milk that could disperse during storage period using by ascorbic acid as a stabilizer and fortifier of vitamin C (Seo et al., 2011). To explore antibacteriocide action, a nanochitosan study was also conducted to observe this action using lactic acid bacteria in yogurt. The result of this study showed that NPC-added yogurt contained lactic acid bacteria from $4.75 \times 10^{8}$ to $9.70 \times 10^{8} \mathrm{CFU} / \mathrm{mL}$ during storage at $4^{\circ} \mathrm{C}$ for $20 \mathrm{~d}$, which was not significantly different from control $(p>0.05)$ (Seo et al., 2009).

Maribo cheese is one of the representative semi-hard 

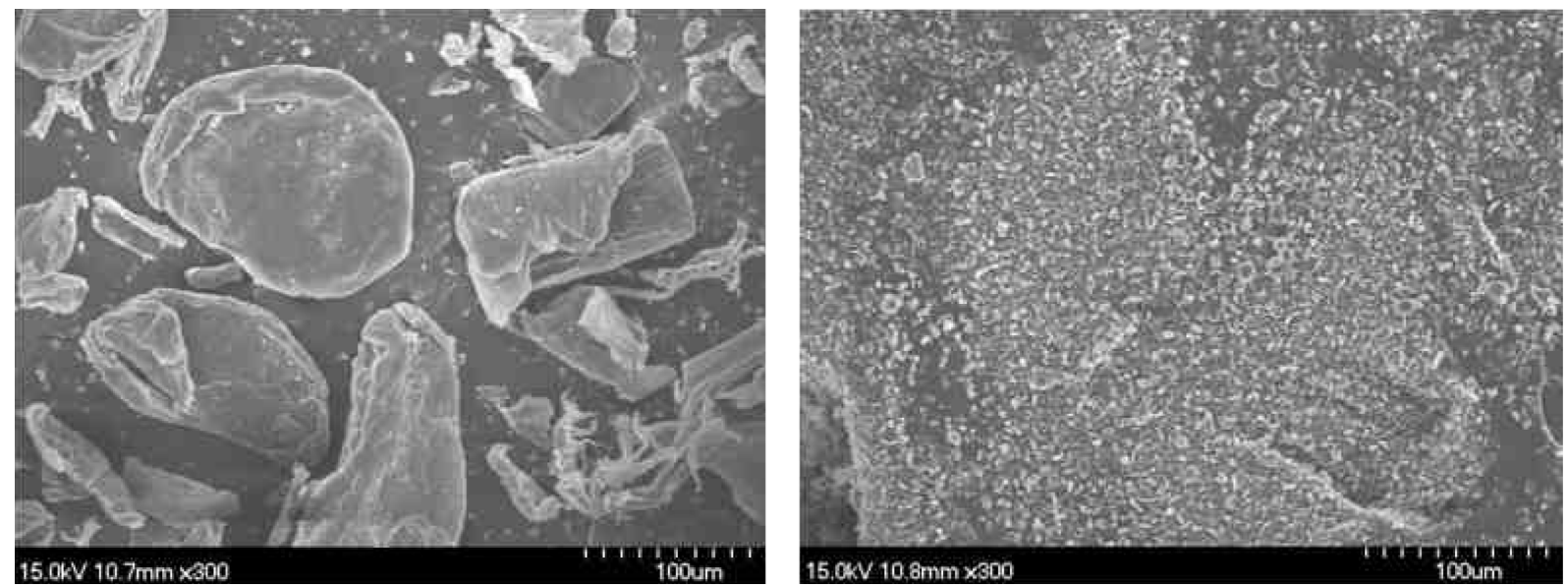

Fig. 1. Scanning electron microscope images for powdered chitosan and nanopowdered chitosan.

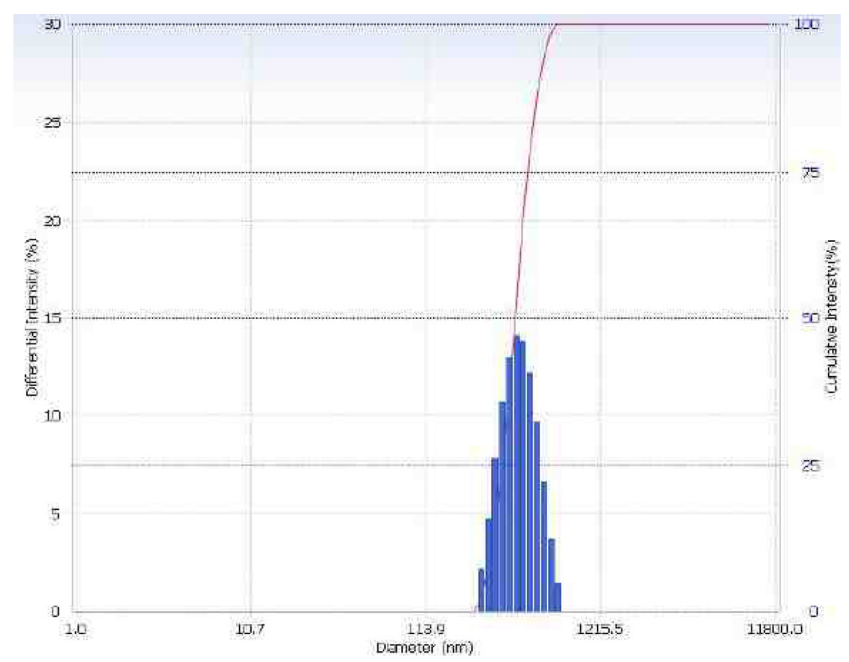

Fig. 2. Particle size of nanopowdered chitosan.

cheeses which has been made mainly in Denmark, and it resembles the various Latin America cheeses, such as chanco and paipa in Colombia and prato in Brazil (Brito et al., 2000). Since nanotechnology is quite new area in food science, there are almost no reports in the literature on the production of NPC-added Maribo cheese. Therefore, the objective of this study was focused on the physicochemical and sensory properties of cheese added with NPC during ripening.

\section{Materials and Methods}

\section{Materials}

Raw milk to manufacture Maribo cheese was obtained from an animal farm affiliated with Sunchon National University (Sunchon, Korea). Powdered chitosan was obtained from Samsung Chitopia (Seoul, Korea) and milled to NPC by dry milling method in Apexel Co. (Pohang, Korea). The average particle sizes of PC and NPC measured were about $150 \mu \mathrm{m}$ (as measured by scanning electron microscope) and about $562 \mathrm{~nm}$ in diameter (as measured by the particle size analyzer), respectively (Fig. 1 and 2) (Seo et al., 2009).

\section{Manufacture of NCMC}

Maribo cheese was prepared as described previously (Robinson, 1995) with some modification. Different concentrations of NPC ( 0.2 and $0.5 \%)$ were added at the curd after second whey drainage. After manufacturing, pressed cheese were dried for $2 \mathrm{~d}$ and ripened at $14^{\circ} \mathrm{C}$ with a relative humidity of $90-95 \%$ for 6 mon.

\section{Analysis of chemical composition and yield of cheese}

Cheese was analyzed for moisture, fat, and protein using methods of Association of Official Analytical Chemists (AOAC, 2000). Cheese yield was determined as wt. cheese $\times 100 /$ wt. milk.

\section{Analysis of short-chain fatty acids}

Cheese samples $(1 \mathrm{~g})$ were taken periodically, stored for $0,1,2,3,4,5$, and 6 mon, extracted with diethyl ether and hexane for $2 \mathrm{~h}$, and eluted through a $10 \mathrm{~mm}$ inner diameter glass column containing neutral alumina as described by Kwak et al. (1990). A Hewlett-Packard Model 5880A GC equipped with a flame ionization detector was used. The quantitation of short-chain fatty acid (SCFA) was achieved using a $15 \mathrm{~m} \times 0.53 \mathrm{~mm}$ inner diameter Nukol fused-silica capillary column (Supleco Inc., Bellefonte, PA, USA). The GC was operated with nitrogen carrier gas at $30 \mathrm{~mL} / \mathrm{min}$, hydrogen gas $30 \mathrm{~mL} / \mathrm{min}$, and air at $300 \mathrm{~mL} / \mathrm{min}$. The column oven was programmed at the 
initial holding for $1 \mathrm{~min}$ at $60^{\circ} \mathrm{C}$ and the first level holding to $170^{\circ} \mathrm{C}$ at $5^{\circ} \mathrm{C} / \mathrm{min}$ for $10 \mathrm{~min}$ and holding for $20 \mathrm{~min}$. Both temperatures for injector and detector were $220^{\circ} \mathrm{C}$. Each FFA was identified by the retention time of standard.

\section{Color measurements}

Color values of control and NCMC were measured using a colorimeter (Minolta CT-310, Tokyo, Japan) after calibrating its original value with a standard plate. Measured $\mathrm{L}^{*}, \mathrm{a}^{*}$, and $\mathrm{b}^{*}$ values were used as indicators of lightness, redness, and yellowness, respectively. All samples were measured ten times.

\section{Texture profile analysis}

Texture profile was measured using a TMS-Pro Texture Analyzer (Food Technology Co., VA, USA) with a crosshead of $5 \mathrm{~cm} / \mathrm{min}$ and a chart recorder speed of $10 \mathrm{~cm} /$ min. Cube-shaped samples $\left(1.5 \mathrm{~cm}^{3}\right)$ were prepared. The basic characteristics, such as hardness $(\mathrm{N})$, cohesiveness $\left(\mathrm{J}^{3}\right)$, gumminess $(\mathrm{N})$, springiness $\left(\mathrm{m} \times 10^{-3}\right)$, and chewiness $\left(\mathrm{J} \times 10^{-3}\right)$, were obtained.

\section{Sensory attribute}

Sensory evaluation was conducted by ten-trained sensory panelists. Flavor, taste, and texture were investigated on a 7 -point scale $(1=$ very weak, $4=$ moderate and $7=$ very strong). Overall acceptability was also evaluated on a 7 -point scale $(1=$ dislike very much, $4=$ neither like nor dislike and $7=$ like very much). Samples were presented in randomly coded glass plates.

\section{Statistical analysis}

All statistical analyses were performed using SAS version 9.0 (SAS Institute Inc., USA). Analysis of variance was performed using the general linear models procedure to determine significant differences among the samples. Means were compared by using Fisher's least significant difference procedure. Significance was defined at the $5 \%$ level.

\section{Results and Discussion}

\section{Chemical composition}

The composition of NCMC is shown in Table 1. Moisture contents were not significantly different among all treatments with $36.95 \%$ in control cheese $(p>0.05)$. However, protein decreased significantly with increasing added chitosans from $24.25 \%$ in control to $22.91 \%$ in $0.5 \%$ NCMC $(p<0.05)$. Fat contents were not significantly affected from the addition of chitosan $(p>0.05)$. Ash contents increased with increasing chitosan concentrations of PCMC and NCMC. $0.5 \%$ NCMC had the highest ash contents among the samples (4.00\%). Addition of nanochitosan may contribute to decreasing of nitrogen contents and increasing of ash contents. Cichoscki et al. (2002) reported that chemical composition, such as protein, fat, and ash of Prato cheese which is one of Brazilian semi-hard cheeses wasn't significantly different during $60 \mathrm{~d}$ ripening.

\section{SCFA}

The production of SCFAs $\left(\mathrm{C}_{4}\right.$ through $\left.\mathrm{C}_{10}\right)$ is important in cheese ripening (Lin and Jeon, 1987). They contribute indirectly to cheese flavor by acting as precursors for the production of volatile flavor compounds (Mcsweeney, 2003). The production of SCFA in NCMC during 6 mon ripening at $14^{\circ} \mathrm{C}$ was shown in Table 2. SCFAs in NCMC and PCMC were increased during ripening. Release of butyric acid $\left(\mathrm{C}_{4}\right)$ contributed to the increase in total amount of SCFAs until 1 mon. After that, capric acid $\left(\mathrm{C}_{10}\right)$ influenced the increase of total SCFAs. $\mathrm{C}_{4}$ of all treatments was increased slightly during 3 mon ripening with no significant difference ( $p>0.05$ ). At 4 mon, $\mathrm{C}_{4}$ of all the samples was dramatically increased by almost 2 times. After 4 mon, $\mathrm{C}_{4}$ of $\mathrm{NCMC}$ and PCMC was observed to have significantly more production than that of control $(p<0.05)$. After the ripening, $0.2 \% \mathrm{NCMC}$ and

Table 1. Composition of nanopowdered chitosan-added Maribo cheese

\begin{tabular}{ccccc}
\hline \hline Treatment $(\% \mathrm{w} / \mathrm{w})$ & \multicolumn{1}{c}{ Moisture } & \multicolumn{1}{c}{ Protein } & Ash \\
\hline Control & $36.95 \pm 0.6^{\mathrm{A}}$ & $24.25 \pm 0.3^{\mathrm{AB}}$ & $35.27 \pm 0.3^{\mathrm{BC}}$ & $3.33 \pm 0.04^{\mathrm{D}}$ \\
$0.2 \mathrm{PCMC}$ & $36.22 \pm 1.1^{\mathrm{A}}$ & $24.25 \pm 0.5^{\mathrm{A}}$ & $36.88 \pm 0.6^{\mathrm{A}}$ & $3.57 \pm 0.02^{\mathrm{C}}$ \\
$0.5 \mathrm{PCMC}$ & $36.01 \pm 0.3^{\mathrm{A}}$ & $23.03 \pm 0.3^{\mathrm{BC}}$ & $33.97 \pm 0.5^{\mathrm{C}}$ & $3.60 \pm 0.00^{\mathrm{C}}$ \\
$0.2 \mathrm{NCMC}$ & $36.21 \pm 0.6^{\mathrm{A}}$ & $23.31 \pm 0.5^{\mathrm{ABC}}$ & $35.62 \pm 0.5^{\mathrm{AB}}$ & $3.74 \pm 0.04^{\mathrm{B}}$ \\
$0.5 \mathrm{NCMC}$ & $36.88 \pm 0.4^{\mathrm{A}}$ & $22.91 \pm 0.2^{\mathrm{C}}$ & $35.34 \pm 0.3^{\mathrm{BC}}$ & $4.00 \pm 0.00^{\mathrm{A}}$ \\
\hline
\end{tabular}

Means within a column with different letters are significantly different at $p<0.05$

Control: untreated Maribo cheese.

PCMC: powdered chitosan-added Maribo cheese.

NCMC: nanopowdered chitosan-added Maribo cheese. 
Table 2. Production of short-chain fatty acid in nanopowdered chitosan-added Maribo cheese during ripening at $14^{\circ} \mathrm{C}$ for 6 mon

\begin{tabular}{|c|c|c|c|c|c|c|}
\hline \multirow{2}{*}{$\begin{array}{l}\text { Ripening period } \\
\text { (mon) }\end{array}$} & \multirow{2}{*}{$\begin{array}{l}\text { Treatment } \\
(\% \mathrm{w} / \mathrm{w})\end{array}$} & \multicolumn{5}{|c|}{ SCFA concentration (ppm) } \\
\hline & & $\mathrm{C}_{4}$ & $\mathrm{C}_{6}$ & $\mathrm{C}_{8}$ & $\mathrm{C}_{10}$ & Total \\
\hline \multirow{5}{*}{0} & Control & $5.36 \pm 0.12^{\mathrm{A}}$ & $2.36 \pm 0.18^{\mathrm{A}}$ & $2.85 \pm 0.01^{\mathrm{A}}$ & $3.24 \pm 0.25^{\mathrm{A}}$ & 13.81 \\
\hline & 0.2 PCMC & $5.55 \pm 0.11^{\mathrm{A}}$ & $2.40 \pm 0.23^{\mathrm{A}}$ & $2.81 \pm 0.03^{\mathrm{A}}$ & $3.31 \pm 0.19^{\mathrm{A}}$ & 14.07 \\
\hline & 0.5 PCMC & $5.39 \pm 0.04^{\mathrm{A}}$ & $2.49 \pm 0.14^{\mathrm{A}}$ & $2.82 \pm 0.06^{\mathrm{A}}$ & $3.28 \pm 0.34^{\mathrm{A}}$ & 13.98 \\
\hline & $0.2 \mathrm{NCMC}$ & $5.27 \pm 0.13^{\mathrm{A}}$ & $2.74 \pm 0.13^{\mathrm{A}}$ & $2.43 \pm 0.35^{\mathrm{A}}$ & $3.35 \pm 0.36^{\mathrm{A}}$ & 13.79 \\
\hline & $0.5 \mathrm{NCMC}$ & $5.48 \pm 0.34^{\mathrm{A}}$ & $2.56 \pm 0.14^{\mathrm{A}}$ & $2.76 \pm 0.11^{\mathrm{A}}$ & $3.30 \pm 0.13^{\mathrm{A}}$ & 14.10 \\
\hline \multirow{5}{*}{1} & Control & $5.76 \pm 0.09^{A}$ & $2.70 \pm 0.02^{\mathrm{A}}$ & $3.02 \pm 0.05^{\mathrm{B}}$ & $4.28 \pm 0.04^{\mathrm{A}}$ & 15.76 \\
\hline & 0.2 PCMC & $5.80 \pm 0.08^{\mathrm{A}}$ & $2.74 \pm 0.16^{\mathrm{A}}$ & $3.15 \pm 0.05^{\mathrm{A}}$ & $4.58 \pm 0.04^{\mathrm{A}}$ & 16.27 \\
\hline & 0.5 PCMC & $5.84 \pm 0.05^{\mathrm{A}}$ & $2.78 \pm 0.18^{\mathrm{A}}$ & $3.10 \pm 0.01^{\mathrm{AB}}$ & $4.61 \pm 0.16^{\mathrm{A}}$ & 16.33 \\
\hline & $0.2 \mathrm{NCMC}$ & $5.70 \pm 0.03^{\mathrm{A}}$ & $2.77 \pm 0.09^{\mathrm{A}}$ & $3.03 \pm 0.05^{\mathrm{AB}}$ & $4.52 \pm 0.23^{\mathrm{A}}$ & 16.02 \\
\hline & $0.5 \mathrm{NCMC}$ & $5.81 \pm 0.05^{\mathrm{A}}$ & $2.73 \pm 0.20^{\mathrm{A}}$ & $3.13 \pm 0.04^{\mathrm{AB}}$ & $4.56 \pm 0.09^{\mathrm{A}}$ & 16.23 \\
\hline \multirow{5}{*}{2} & Control & $6.01 \pm 0.08^{A}$ & $3.11 \pm 0.08^{\mathrm{A}}$ & $3.69 \pm 0.09^{A}$ & $10.57 \pm 0.08^{\mathrm{B}}$ & 23.38 \\
\hline & 0.2 PCMC & $5.87 \pm 0.06^{\mathrm{A}}$ & $3.16 \pm 0.06^{\mathrm{A}}$ & $3.71 \pm 0.17^{\mathrm{A}}$ & $12.56 \pm 0.20^{\mathrm{A}}$ & 25.30 \\
\hline & 0.5 PCMC & $5.91 \pm 0.04^{\mathrm{A}}$ & $3.15 \pm 0.03^{\mathrm{A}}$ & $3.65 \pm 0.13^{\mathrm{A}}$ & $12.72 \pm 0.23^{\mathrm{A}}$ & 25.43 \\
\hline & $0.2 \mathrm{NCMC}$ & $5.94 \pm 0.06^{\mathrm{A}}$ & $3.15 \pm 0.09^{\mathrm{A}}$ & $3.74 \pm 0.08^{\mathrm{A}}$ & $12.65 \pm 0.13^{\mathrm{A}}$ & 25.48 \\
\hline & $0.5 \mathrm{NCMC}$ & $5.93 \pm 0.05^{\mathrm{A}}$ & $3.17 \pm 0.03^{\mathrm{A}}$ & $3.76 \pm 0.13^{\mathrm{A}}$ & $12.68 \pm 0.11^{\mathrm{A}}$ & 25.54 \\
\hline \multirow{5}{*}{3} & Control & $6.69 \pm 0.64^{\mathrm{AB}}$ & $3.30 \pm 0.08^{\mathrm{A}}$ & $5.70 \pm 0.25^{\mathrm{A}}$ & $16.49 \pm 1.31^{\mathrm{A}}$ & 32.18 \\
\hline & 0.2 PCMC & $5.83 \pm 0.37^{\mathrm{B}}$ & $3.25 \pm 0.08^{\mathrm{A}}$ & $5.61 \pm 0.34^{\mathrm{A}}$ & $17.70 \pm 1.23^{\mathrm{A}}$ & 32.39 \\
\hline & 0.5 PCMC & $6.26 \pm 0.06^{\mathrm{AB}}$ & $3.55 \pm 0.10^{\mathrm{A}}$ & $5.70 \pm 0.13^{\mathrm{A}}$ & $17.57 \pm 0.68^{\mathrm{A}}$ & 33.08 \\
\hline & $0.2 \mathrm{NCMC}$ & $7.16 \pm 0.47^{\mathrm{AB}}$ & $3.39 \pm 0.16^{\mathrm{A}}$ & $5.82 \pm 0.25^{\mathrm{A}}$ & $17.83 \pm 1.18^{\mathrm{A}}$ & 34.20 \\
\hline & $0.5 \mathrm{NCMC}$ & $7.99 \pm 1.29^{\mathrm{A}}$ & $3.57 \pm 0.23^{\mathrm{A}}$ & $5.80 \pm 0.13^{\mathrm{A}}$ & $17.19 \pm 1.05^{\mathrm{A}}$ & 34.55 \\
\hline \multirow{5}{*}{4} & Control & $11.09 \pm 1.24^{\mathrm{B}}$ & $4.22 \pm 0.08^{\mathrm{C}}$ & $5.89 \pm 0.04^{\mathrm{A}}$ & $18.14 \pm 0.11^{\mathrm{C}}$ & 44.98 \\
\hline & 0.2 PCMC & $13.79 \pm 0.07^{\mathrm{A}}$ & $4.56 \pm 0.08^{\mathrm{A}}$ & $5.74 \pm 0.06^{\mathrm{B}}$ & $18.21 \pm 0.06^{\mathrm{C}}$ & 42.30 \\
\hline & 0.5 PCMC & $14.52 \pm 0.65^{\mathrm{A}}$ & $4.49 \pm 0.10^{\mathrm{AB}}$ & $5.86 \pm 0.06^{\mathrm{AB}}$ & $18.94 \pm 0.35^{\mathrm{AB}}$ & 43.81 \\
\hline & $0.2 \mathrm{NCMC}$ & $13.38 \pm 0.37^{\mathrm{A}}$ & $4.28 \pm 0.16^{\mathrm{BC}}$ & $5.95 \pm 0.03^{\mathrm{A}}$ & $18.40 \pm 0.31^{\mathrm{BC}}$ & 42.01 \\
\hline & $0.5 \mathrm{NCMC}$ & $13.86 \pm 1.12^{\mathrm{A}}$ & $4.50 \pm 0.02^{\mathrm{AB}}$ & $5.92 \pm 0.04^{\mathrm{A}}$ & $19.27 \pm 0.08^{\mathrm{A}}$ & 43.55 \\
\hline \multirow{5}{*}{5} & Control & $13.48 \pm 0.30^{\mathrm{B}}$ & $4.88 \pm 0.10^{\mathrm{A}}$ & $6.11 \pm 0.08^{\mathrm{A}}$ & $20.51 \pm 0.04^{\mathrm{A}}$ & 44.98 \\
\hline & 0.2 PCMC & $16.61 \pm 0.97^{\mathrm{AB}}$ & $4.92 \pm 0.06^{\mathrm{A}}$ & $6.23 \pm 0.06^{\mathrm{A}}$ & $21.62 \pm 0.11^{\mathrm{A}}$ & 49.38 \\
\hline & 0.5 PCMC & $17.19 \pm 0.67^{\mathrm{A}}$ & $5.00 \pm 0.02^{\mathrm{A}}$ & $6.32 \pm 0.35^{\mathrm{A}}$ & $21.38 \pm 0.74^{\mathrm{A}}$ & 49.89 \\
\hline & $0.2 \mathrm{NCMC}$ & $16.16 \pm 0.67^{\mathrm{A}}$ & $4.93 \pm 0.09^{\mathrm{A}}$ & $6.10 \pm 0.01^{\mathrm{A}}$ & $20.68 \pm 0.04^{\mathrm{A}}$ & 47.87 \\
\hline & $0.5 \mathrm{NCMC}$ & $16.52 \pm 1.55^{\mathrm{A}}$ & $5.00 \pm 0.16^{\mathrm{A}}$ & $6.23 \pm 0.11^{\mathrm{A}}$ & $21.15 \pm 0.78^{\mathrm{A}}$ & 48.90 \\
\hline \multirow{5}{*}{6} & Control & $15.18 \pm 1.01^{\mathrm{B}}$ & $6.88 \pm 0.11^{\mathrm{A}}$ & $7.08 \pm 0.23^{\mathrm{A}}$ & $25.13 \pm 0.25^{\mathrm{A}}$ & 53.11 \\
\hline & 0.2 PCMC & $18.09 \pm 0.76^{\mathrm{AB}}$ & $6.95 \pm 0.13^{\mathrm{A}}$ & $7.14 \pm 0.06^{\mathrm{A}}$ & $25.15 \pm 2.35^{\mathrm{A}}$ & 57.33 \\
\hline & 0.5 PCMC & $21.97 \pm 0.92^{\mathrm{A}}$ & $7.08 \pm 0.13^{\mathrm{A}}$ & $7.17 \pm 0.04^{\mathrm{A}}$ & $27.38 \pm 2.64^{\mathrm{A}}$ & 62.78 \\
\hline & $0.2 \mathrm{NCMC}$ & $19.34 \pm 2.61^{\mathrm{AB}}$ & $7.09 \pm 0.30^{\mathrm{A}}$ & $7.16 \pm 0.01^{\mathrm{A}}$ & $27.85 \pm 2.76^{\mathrm{A}}$ & 59.94 \\
\hline & $0.5 \mathrm{NCMC}$ & $20.32 \pm 2.39^{\mathrm{A}}$ & $7.21 \pm 0.27^{\mathrm{A}}$ & $7.20 \pm 0.02^{\mathrm{A}}$ & $26.96 \pm 2.78^{\mathrm{A}}$ & 60.69 \\
\hline
\end{tabular}

Means within a column with different letters are significantly different at $p<0.05$

Control: untreated Maribo cheese.

PCMC: powdered chitosan-added Maribo cheese.

NCMC: nanopowdered chitosan-added Maribo cheese.

control revealed 19.34 and $15.18 \mathrm{ppm}$ in $\mathrm{C}_{4}$, respectively. $\mathrm{C}_{10}$ was also increased during the ripening periods. In 0 and 1 mon, $\mathrm{C}_{10}$ did not show difference among the samples. At 2 mon, $C_{10}$ of all the samples was considerably increased by more than 2 times. NCMC and PCMC had more $\mathrm{C}_{10}$ contents compared to control, while the production of all samples was not significantly different $(p>$ $0.05)$. However, addition of chitosan was not significantly influencial in increasing caproic acid $\left(\mathrm{C}_{6}\right)$ and caprylic acid $\left(\mathrm{C}_{8}\right)$ during ripening period $(p>0.05)$. Both NCMC and PCMC had more $\mathrm{C}_{6}$ and $\mathrm{C}_{8}$ contents than those of control. For total SCFAs, there was no big difference among the samples at 0 mon, however, $0.2 \% \mathrm{NCMC}$ was increased from 13.79 to $59.94 \mathrm{ppm}$ and control was increased from 13.81 to $53.11 \mathrm{ppm}$ during the ripening. Compared to initial, NCMC had higher SCFA concentration than control during the ripening.

\section{Color}

Color of NCMC ripened at $14^{\circ} \mathrm{C}$ for 6 mon was shown in Table $3 . \mathrm{L}^{*}$ value was significantly decreased in all samples during ripening $(p<0.05) .0 .5 \% \mathrm{NCMC}$ had the 
Table 3. Changes in color of nanopowdered chitosan-added Maribo cheese during ripening at $14^{\circ} \mathrm{C}$ for 6 mon

\begin{tabular}{|c|c|c|c|c|c|c|c|c|}
\hline \multirow{2}{*}{$\begin{array}{l}\text { Color } \\
\text { value }\end{array}$} & \multirow{2}{*}{$\begin{array}{l}\text { Treatment } \\
(\% \mathrm{w} / \mathrm{w})\end{array}$} & \multicolumn{7}{|c|}{ Ripening period (mon) } \\
\hline & & 0 & 1 & 2 & 3 & 4 & 5 & 6 \\
\hline \multirow{5}{*}{$\mathrm{L}^{*}$} & Control & $85.74 \pm 0.95^{\mathrm{Aa}}$ & $82.95 \pm 0.60^{\mathrm{Ab}}$ & $81.87 \pm 0.65^{\mathrm{Ac}}$ & $81.40 \pm 0.94^{\mathrm{Ac}}$ & $80.04 \pm 1.36^{\mathrm{Bd}}$ & $79.25 \pm 1.80^{\mathrm{Ad}}$ & $77.20 \pm 0.93^{\mathrm{Be}}$ \\
\hline & $0.2 \mathrm{PC}$ & $85.86 \pm 0.38^{\mathrm{Aa}}$ & $82.81 \pm 0.60^{\mathrm{Ab}}$ & $82.30 \pm 0.60^{\mathrm{Ab}}$ & $81.49 \pm 1.07^{\mathrm{Ac}}$ & $81.26 \pm 0.89^{\mathrm{Ac}}$ & $79.98 \pm 1.05^{\mathrm{Ad}}$ & $78.47 \pm 0.73^{\mathrm{Ae}}$ \\
\hline & $0.5 \mathrm{PCMC}$ & $85.95 \pm 0.53^{\mathrm{Aa}}$ & $82.97 \pm 0.73^{\mathrm{Ab}}$ & $82.06 \pm 0.97^{\mathrm{Ac}}$ & $81.46 \pm 0.49^{\mathrm{Acd}}$ & $80.92 \pm 1.06^{\mathrm{ABd}}$ & $79.91 \pm 1.79^{\mathrm{Ae}}$ & $77.31 \pm 0.49^{\mathrm{Bf}}$ \\
\hline & $0.2 \mathrm{NCMC}$ & $86.06 \pm 0.67^{\mathrm{Aa}}$ & $82.98 \pm 0.55^{\mathrm{Ab}}$ & $82.25 \pm 0.41^{\mathrm{Abc}}$ & $81.41 \pm 1.71^{\mathrm{Acd}}$ & $81.04 \pm 0.84^{\mathrm{ABd}}$ & $79.65 \pm 0.85^{\mathrm{Ae}}$ & $77.49 \pm 1.14^{\mathrm{Bf}}$ \\
\hline & $0.5 \mathrm{NCMC}$ & $85.92 \pm 0.48^{\mathrm{Aa}}$ & $82.78 \pm 0.54^{\mathrm{Ab}}$ & $80.76 \pm 0.56^{\mathrm{Bc}}$ & $80.64 \pm 0.79^{A c}$ & $80.31 \pm 1.28^{\mathrm{ABc}}$ & $78.25 \pm 0.50^{\mathrm{Ad}}$ & $75.86 \pm 0.57^{\mathrm{Ce}}$ \\
\hline \multirow{5}{*}{$a^{*}$} & Control & $2.99 \pm 0.17^{\mathrm{Ae}}$ & $3.07 \pm 0.02^{\mathrm{Ad}}$ & $3.12 \pm 0.02^{\mathrm{ABcd}}$ & $3.19 \pm 0.04^{\mathrm{Abc}}$ & $3.19 \pm 0.04^{\mathrm{Abc}}$ & $3.22 \pm 0.10^{\mathrm{Ab}}$ & $3.58 \pm 0.05^{\mathrm{Aa}}$ \\
\hline & $0.2 \mathrm{PCMC}$ & $2.93 \pm 0.10^{\mathrm{ABd}}$ & $3.09 \pm 0.05^{\mathrm{Ac}}$ & $3.14 \pm 0.07^{\mathrm{ABbc}}$ & $3.14 \pm 0.10^{\mathrm{Abc}}$ & $3.15 \pm 0.02^{\mathrm{ABbc}}$ & $3.20 \pm 0.14^{\mathrm{Ab}}$ & $3.41 \pm 0.09^{\mathrm{Ba}}$ \\
\hline & 0.5 PCMC & $2.92 \pm 0.09^{\mathrm{ABd}}$ & $3.61 \pm 0.05^{\mathrm{Ac}}$ & $3.15 \pm 0.06^{\mathrm{Abc}}$ & $3.14 \pm 0.05^{\mathrm{Ac}}$ & $3.15 \pm 0.15^{\mathrm{ABbc}}$ & $3.25 \pm 0.21^{\mathrm{Ab}}$ & $3.39 \pm 0.04^{\mathrm{BCa}}$ \\
\hline & $0.2 \mathrm{NCMC}$ & $2.86 \pm 0.04^{\mathrm{Bc}}$ & $3.00 \pm 0.10^{\mathrm{Bb}}$ & $3.07 \pm 0.09^{\mathrm{BCb}}$ & $3.09 \pm 0.04^{\mathrm{Bb}}$ & $3.10 \pm 0.02^{\mathrm{Bb}}$ & $3.21 \pm 0.12^{\mathrm{Aa}}$ & $3.27 \pm 0.21^{\mathrm{BCa}}$ \\
\hline & $0.5 \mathrm{NCMC}$ & $2.57 \pm 0.13^{\mathrm{Ce}}$ & $2.84 \pm 0.03^{\mathrm{Cd}}$ & $3.03 \pm 0.11^{\mathrm{Cc}}$ & $3.05 \pm 0.02^{\mathrm{Bc}}$ & $3.09 \pm 0.08^{\mathrm{Bbc}}$ & $3.19 \pm 0.15^{\mathrm{Aab}}$ & $3.26 \pm 0.24^{\mathrm{Ca}}$ \\
\hline \multirow{5}{*}{$\mathrm{b}^{*}$} & Control & $10.93 \pm 0.33^{\mathrm{Ad}}$ & $12.06 \pm 0.11^{\mathrm{ABc}}$ & $12.62 \pm 0.22^{\mathrm{Bb}}$ & $12.69 \pm 0.23^{\mathrm{Bab}}$ & $12.85 \pm 0.53^{\mathrm{Bab}}$ & $12.86 \pm 0.05^{\mathrm{Cab}}$ & $12.93 \pm 0.12^{\mathrm{Ba}}$ \\
\hline & $0.2 \mathrm{PCMC}$ & $10.91 \pm 0.27^{\mathrm{Af}}$ & $11.89 \pm 0.32^{\mathrm{Be}}$ & $12.46 \pm 0.17^{\mathrm{Bd}}$ & $12.55 \pm 0.26^{\mathrm{Bcd}}$ & $12.71 \pm 0.22^{\mathrm{Bc}}$ & $12.93 \pm 0.16^{\mathrm{Cb}}$ & $13.25 \pm 0.14^{\mathrm{Aa}}$ \\
\hline & $0.5 \mathrm{PCMC}$ & $11.05 \pm 0.39^{\mathrm{Ad}}$ & $12.30 \pm 0.35^{\mathrm{ABc}}$ & $12.94 \pm 0.58^{\mathrm{Ab}}$ & $13.07 \pm 0.20^{\mathrm{Aab}}$ & $13.14 \pm 0.20^{\mathrm{Aab}}$ & $13.17 \pm 0.24^{\mathrm{ABab}}$ & $13.42 \pm 0.53^{\mathrm{Aa}}$ \\
\hline & $0.2 \mathrm{NCMC}$ & $11.11 \pm 0.23^{\mathrm{Ad}}$ & $12.15 \pm 0.29^{\mathrm{ABc}}$ & $12.33 \pm 0.24^{\mathrm{Bc}}$ & $12.67 \pm 0.08^{\mathrm{Bb}}$ & $12.98 \pm 0.19^{\mathrm{ABa}}$ & $13.01 \pm 0.35^{\mathrm{BCa}}$ & $13.20 \pm 0.18^{\mathrm{Aa}}$ \\
\hline & $0.5 \mathrm{NCMC}$ & $10.87 \pm 0.10^{\mathrm{Af}}$ & $12.06 \pm 0.19^{\mathrm{ABe}}$ & $13.01 \pm 0.20^{\mathrm{Ad}}$ & $13.13 \pm 0.15^{\text {Acd }}$ & $13.17 \pm 0.05^{\mathrm{Abc}}$ & $13.28 \pm 0.09^{\mathrm{Aab}}$ & $13.39 \pm 0.17^{\mathrm{Aa}}$ \\
\hline
\end{tabular}

Means with different superscripts in a row (a-f) and column (A-C) are significant at $p<0.05$.

Control: untreated Maribo cheese.

PC: powdered chitosan-added Maribo cheese.

NPC: nanopowdered chitosan-added Maribo cheese.

Table 4. Texture properties of nanopowdered chitosan-added Maribo cheese during ripeing at $14^{\circ} \mathrm{C}$ for 6 mon

\begin{tabular}{|c|c|c|c|c|c|c|c|c|}
\hline \multirow{2}{*}{$\begin{array}{c}\text { Texture } \\
\text { description }\end{array}$} & \multirow{2}{*}{$\begin{array}{l}\text { Treatment } \\
(\% \mathrm{w} / \mathrm{w})\end{array}$} & \multicolumn{7}{|c|}{ Ripening period (mon) } \\
\hline & & 0 & 1 & 2 & 3 & 4 & 5 & 6 \\
\hline \multirow{5}{*}{$\begin{array}{l}\text { Hardness } \\
\left(\mathrm{N}^{2}\right)\end{array}$} & Control & $14.97 \pm 0.61^{\mathrm{Aa}}$ & $9.76 \pm 1.21^{\mathrm{Ab}}$ & $47 \pm 0.94^{\mathrm{Ab}}$ & $7.41 \pm 0.47^{\mathrm{Bc}}$ & $7.32 \pm 0.80^{\mathrm{Bc}}$ & $7.19 \pm 1.22^{\mathrm{Bc}}$ & $7.15 \pm 0.66^{\mathrm{Bc}}$ \\
\hline & 0.2 PCMC & $14.99 \pm 0.62^{\mathrm{Aa}}$ & $9.92 \pm 0.90^{\mathrm{Ab}}$ & $.87 \pm 1.09^{\mathrm{Ab}}$ & $9.33 \pm 1.02^{\mathrm{Ab}}$ & $9.34 \pm 0.48^{\mathrm{Ab}}$ & $9.27 \pm 0.71^{\mathrm{Ab}}$ & $9.14 \pm 0.09^{\mathrm{Ab}}$ \\
\hline & 0.5 PCMC & $15.08 \pm 0.51^{\mathrm{Aa}}$ & $10.26 \pm 0.82^{\mathrm{Ab}}$ & $10.05 \pm 1.41^{\mathrm{Ab}}$ & $9.71 \pm 0.60^{\mathrm{Ab}}$ & $9.51 \pm 0.74^{\mathrm{Ab}}$ & $9.42 \pm 0.51^{\mathrm{Ab}}$ & $9.23 \pm 1.48^{\mathrm{Ab}}$ \\
\hline & $0.2 \mathrm{~N}$ & $14.98 \pm 0.30^{\mathrm{Aa}}$ & $9.48 \pm 0.80^{\mathrm{Ab}}$ & $9.18 \pm 0.62^{\mathrm{Abc}}$ & $8.89 \pm 0.98^{\mathrm{Abc}}$ & $8.43 \pm 0.81^{\mathrm{ABc}}$ & $8.38 \pm 0.62^{\mathrm{Ac}}$ & $8.19 \pm 0.49^{\mathrm{ABc}}$ \\
\hline & MC & $15.04 \pm 1.16^{\mathrm{Aa}}$ & $9.96 \pm 1.00^{\mathrm{Ab}}$ & $9.64 \pm 0.99^{\mathrm{Ab}}$ & $9.30 \pm 1.11^{\mathrm{Ab}}$ & $9.26 \pm 1.42^{\mathrm{Ab}}$ & $9.23 \pm 0.86^{\mathrm{Ab}}$ & $9.20 \pm 0.12^{\mathrm{Ab}}$ \\
\hline \multirow{5}{*}{$\begin{array}{c}\text { Cohesiveness } \\
\qquad\left(\mathrm{J}^{3}\right)\end{array}$} & l & $0.78 \pm 0.02^{\mathrm{Aa}}$ & $0.73 \pm 0.02^{\mathrm{Ab}}$ & $0.69 \pm 0.01^{\mathrm{Ad}}$ & $0.71 \pm 0.01^{\mathrm{Ac}}$ & $0.70 \pm 0.01^{\text {Acd }}$ & $0.68 \pm 0.01^{\text {Ade }}$ & $0.67 \pm 0.01^{\mathrm{Ae}}$ \\
\hline & 0.2 PCMC & $0.78 \pm 0.01^{\mathrm{Aa}}$ & $0.73 \pm 0.02^{\mathrm{Ab}}$ & $0.68 \pm 0.02^{\mathrm{Ac}}$ & $0.68 \pm 0.04^{\mathrm{Bcd}}$ & $0.66 \pm 0.02^{\mathrm{Bcd}}$ & $0.66 \pm 0.02^{\mathrm{Bd}}$ & $0.65 \pm 0.01^{\mathrm{Ad}}$ \\
\hline & 0.5 PCMC & $0.78 \pm 0.02^{\mathrm{Aa}}$ & $0.74 \pm 0.02^{\mathrm{Ab}}$ & $0.68 \pm 0.02^{\mathrm{Ac}}$ & $0.69 \pm 0.01^{\mathrm{ABc}}$ & $0.69 \pm 0.02^{\mathrm{Ac}}$ & $0.67 \pm 0.01^{\mathrm{ABcd}}$ & $0.64 \pm 0.03^{\mathrm{Ad}}$ \\
\hline & 0 & $0.79 \pm 0.02^{\mathrm{Aa}}$ & $0.73 \pm 0.01^{\mathrm{Ab}}$ & $0.69 \pm 0.02^{\mathrm{Ac}}$ & $0.67 \pm 0.01^{\mathrm{Bcd}}$ & $0.69 \pm 0.01^{\mathrm{Ac}}$ & $0.68 \pm 0.02^{\text {Acd }}$ & $0.66 \pm 0.01^{\mathrm{Ad}}$ \\
\hline & $0.5 \mathrm{~N}$ & $0.79 \pm 0.02^{\mathrm{Aa}}$ & $0.74 \pm 0.01^{\mathrm{Ab}}$ & $0.70 \pm 0.02^{\mathrm{Ac}}$ & $0.69 \pm 0.01^{\mathrm{ABcd}}$ & $0.69 \pm 0.01^{\text {Acd }}$ & $0.67 \pm 0.02^{\mathrm{ABde}}$ & $0.67 \pm 0.02^{\mathrm{Ae}}$ \\
\hline \multirow{5}{*}{$\begin{array}{l}\text { Gumminess } \\
\text { (N) }\end{array}$} & pl & $11.39 \pm 0.3$ & $7.78 \pm 0.49^{\mathrm{Ab}}$ & $7.46 \pm 0.31^{\mathrm{Ab}}$ & $5.28 \pm 0.31^{\mathrm{Bc}}$ & $5.26 \pm 1.08^{\mathrm{Bc}}$ & $5.12 \pm 1.21^{\mathrm{Bc}}$ & $5.05 \pm 0.16^{\mathrm{Bc}}$ \\
\hline & $0.2 \mathrm{P}$ & $11.50 \pm 0.44^{\mathrm{Aa}}$ & $7.19 \pm 0.52^{\mathrm{Ab}}$ & $7.01 \pm 0.32^{\mathrm{Ab}}$ & $6.97 \pm 0.67^{\mathrm{Ab}}$ & $6.87 \pm 0.43^{\mathrm{Abc}}$ & $6.57 \pm 0.46^{\mathrm{Abc}}$ & $6.26 \pm 0.52^{\mathrm{Ac}}$ \\
\hline & $0.5 \mathrm{~F}$ & $11.52 \pm 0.46^{\mathrm{Aa}}$ & $7.68 \pm 0.80^{\mathrm{Ab}}$ & $7.22 \pm 0.51^{\mathrm{Abc}}$ & $6.99 \pm 0.22^{\mathrm{Abc}}$ & $6.80 \pm 0.76^{\mathrm{Ac}}$ & $6.81 \pm 0.10^{\mathrm{Ac}}$ & $6.53 \pm 0.65^{\mathrm{Ac}}$ \\
\hline & MC & $0.35^{\mathrm{Aa}}$ & & $7.06 \pm 0.61^{\mathrm{Ab}}$ & $\pm 0.55^{\mathrm{Ab}}$ & $7.01 \pm 1.2$ & $0.92 \pm 0.29$ & $6.67 \pm 0.45^{\mathrm{Ab}}$ \\
\hline & MC & $11.46 \pm 0.7$ & $7.51 \pm 1.11^{\mathrm{Ab}}$ & $7.37 \pm 0.52^{\mathrm{Ab}}$ & $7.00 \pm 0.36^{\mathrm{Ab}}$ & $6.96 \pm 0.69^{\mathrm{Ab}}$ & $6.82 \pm 0.57^{\mathrm{Ab}}$ & $6.70 \pm 0.69^{\mathrm{Ab}}$ \\
\hline \multirow{5}{*}{$\begin{array}{l}\text { Springiness } \\
\left(\mathrm{m} \times 10^{-3}\right)\end{array}$} & 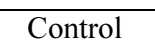 & $0.79 \pm 0.01^{\mathrm{Aa}}$ & $0.75 \pm 0.03^{\mathrm{Aa}}$ & $0.75 \pm 0.08^{\mathrm{Aa}}$ & $0.70 \pm 0.01^{\mathrm{Ab}}$ & $0.70 \pm 0.02^{\mathrm{Ab}}$ & $0.68 \pm 0.02^{\mathrm{Ab}}$ & $0.68 \pm 0.03^{\mathrm{Ab}}$ \\
\hline & 0.2 PCMC & $0.80 \pm 0.11^{\mathrm{Aa}}$ & $0.73 \pm 0.02^{\text {Aab }}$ & $0.73 \pm 0.07^{\mathrm{Aab}}$ & $0.71 \pm 0.02^{\mathrm{Ab}}$ & $0.70 \pm 0.02^{\mathrm{Ab}}$ & $0.69 \pm 0.04^{\mathrm{Ab}}$ & $0.66 \pm 0.03^{\mathrm{Ab}}$ \\
\hline & 0.5 PCMC & $0.85 \pm 0.12^{\mathrm{Aa}}$ & $0.77 \pm 0.12^{\mathrm{Aab}}$ & $0.76 \pm 0.11^{\mathrm{Aabc}}$ & $0.72 \pm 0.03^{\mathrm{Abc}}$ & $0.69 \pm 0.02^{\mathrm{Abc}}$ & $0.65 \pm 0.03^{\mathrm{Ac}}$ & $0.65 \pm 0.03^{\mathrm{Ac}}$ \\
\hline & $0.2 \mathrm{~N}$ & $0.79 \pm 0.14^{\mathrm{Aa}}$ & $0.74 \pm 0.02^{\text {Aab }}$ & $0.73 \pm 0.04^{\mathrm{Aab}}$ & $0.71 \pm 0.01^{\mathrm{Aab}}$ & $0.69 \pm 0.02^{\mathrm{Ab}}$ & $0.67 \pm 0.02^{\mathrm{Ab}}$ & $0.67 \pm 0.03^{\mathrm{Ab}}$ \\
\hline & $0.5 \mathrm{NCMC}$ & $0.83 \pm 0.10^{\mathrm{Aa}}$ & $0.78 \pm 0.10^{\mathrm{Aab}}$ & $0.78 \pm 0.11^{\text {Aab }}$ & $0.72 \pm 0.04^{\mathrm{Abc}}$ & $0.69 \pm 0.03^{\mathrm{Abc}}$ & $0.66 \pm 0.04^{\mathrm{Ac}}$ & $0.66 \pm 0.02^{\mathrm{Ac}}$ \\
\hline \multirow{5}{*}{$\begin{array}{l}\text { Chewiness } \\
\left(\mathrm{J}^{\times} \times 10^{-3}\right)\end{array}$} & 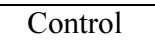 & $9.42 \pm 0.38^{\mathrm{Aa}}$ & $5.13 \pm 0.58^{\mathrm{Ab}}$ & $5.08 \pm 0.39^{\mathrm{Ab}}$ & $5.04 \pm 0.91^{\mathrm{Ab}}$ & $4.97 \pm 0.21^{\mathrm{Ab}}$ & $4.91 \pm 0.29^{\mathrm{Ab}}$ & $4.85 \pm 0.42^{\mathrm{Ab}}$ \\
\hline & 0.2 PCMC & $9.64 \pm 0.97^{\mathrm{Aa}}$ & $5.28 \pm 0.52^{\mathrm{Ab}}$ & $5.15 \pm 0.26^{\mathrm{Ab}}$ & $5.11 \pm 0.74^{\mathrm{Ab}}$ & $5.06 \pm 0.30^{\mathrm{Ab}}$ & $4.98 \pm 0.40^{\mathrm{Ab}}$ & $4.87 \pm 0.34^{\mathrm{Ab}}$ \\
\hline & $0.5 \mathrm{~F}$ & $9.82 \pm 1.10^{\mathrm{Aa}}$ & $5.53 \pm 0.74^{\mathrm{Ab}}$ & $5.31 \pm 0.59^{\mathrm{Ab}}$ & $5.19 \pm 0.74^{\mathrm{Ab}}$ & $5.04 \pm 0.25^{\mathrm{Ab}}$ & $4.97 \pm 0.57^{\mathrm{Ab}}$ & $4.88 \pm 0.14^{\mathrm{Ab}}$ \\
\hline & $0.2 \mathrm{NCMC}$ & $9.52 \pm 1.81^{\mathrm{Aa}}$ & $5.15 \pm 0.84^{\mathrm{Ab}}$ & $5.04 \pm 0.37^{\mathrm{Ab}}$ & $5.01 \pm 0.43^{\mathrm{Ab}}$ & $4.82 \pm 0.48^{\mathrm{Ab}}$ & $4.78 \pm 0.21^{\mathrm{Ab}}$ & $4.80 \pm 0.39^{\mathrm{Ab}}$ \\
\hline & $0.5 \mathrm{NCMC}$ & $9.71 \pm 0.38^{\mathrm{Aa}}$ & $5.38 \pm 0.35^{\mathrm{Ab}}$ & $5.28 \pm 0.47^{\mathrm{Abc}}$ & $5.22 \pm 0.20^{\mathrm{Abc}}$ & $5.04 \pm 0.12^{\mathrm{Abc}}$ & $4.91 \pm 0.29^{\mathrm{Abc}}$ & $4.85 \pm 0.48^{\mathrm{Ac}}$ \\
\hline
\end{tabular}

Means with different superscripts in a row (a-e) and column (A-B) are significant at $p<0.05$.

Control $=$ Maribo cheese.

PC: powdered chitosan-added Maribo cheese.

NPC: nanopowdered chitosan-added Maribo cheese. 
lowest $\mathrm{L}^{*}$ value after ripening (75.86). $0.2 \% \mathrm{NCMC}$ was decreased from 86.06 to 77.49 and this decreasing trend was similar to the control. Concentration of chitosan affected decreasing of lightness. However, $a^{*}$ and $b^{*}$ values of all samples were increased. $a^{*}$ value of chitosan-added cheese was lower than that of control, whereas $b^{*}$ value of chitosan-added cheese was higher than that of control. $a^{*}$ value was decreased with increasing chitosan concentrations. Under the same concentration of chitosan-added cheese, PCMC had significantly high $b^{*}$ values than NCMC. $b *$ values of $0.2 \% \mathrm{NCMC}$ and $0.5 \%$ NCMC were 13.20 and 13.39, respectively. As chitosan concentration increased, $b^{*}$ value increased both in NCMC and PCMC.

Rohm et al. (1996) reported that Emmental cheese also had similar trends in specificity that $\mathrm{L}^{*}$ value decreased but $\mathrm{a}^{*}$ and $\mathrm{b}^{*}$ values increased during $120 \mathrm{~d}$ ripening. According to Seo et al. (2011), nanochitosan solution-added milk samples significantly increased $b *$ value but the addition of NPC did not significantly affect $\mathrm{L}^{*}$ value of nanochitosan-added milk.

\section{Texture analysis}

Textural properties of NCMC during 6 mon ripening stored at $14^{\circ} \mathrm{C}$ are presented in Table 4 . Hardness dec- reased significantly in all samples during the ripening particularly at 1 mon $(p<0.05)$. After 3 mon, NCMC and PCMC had significantly higher hardness compared to control $(p<0.05)$. During the ripening, $0.5 \% \mathrm{NCMC}$ and control decreased from 15.04 to $9.20 \mathrm{~N}^{2}$ and from 14.97 to $7.15 \mathrm{~N}^{2}$, respectively. PCMC was harder than NCMC at each ripening period. Hardness of cheese was decreased because proteolytic breakdown of $\alpha_{s 1}$-casein into peptides causes cheese to soften and lose structural integrity with time (Fox, 1989). Cohesiveness and gumminess were also decreased during the ripening in all samples. Cohesiveness was not significantly different among the samples $(p<0.05)$; however, cohesiveness of NCMC was higher than that of PCMC. After 3 mon, gumminess of control had significantly lower values than that of NCMC and PCMC $(p<0.05)$. NCMC was observed to have more gumminess than PCMC after 4 mon. Springiness and chewiness did not show any significant change by addition of chitosan in cheese during 6 mon ripening $(p>0.05)$. Springiness was slightly decreased during ripening and chewiness was decreased by almost 2 times at 1 mon in all samples. Tunick and Van Hekken (2000) reported that most of the cheeses became less cohesive with increasing age and the values for chewiness usually decreased with stor-

Table 5. Sensory properties of nanopowdered chitosan-added Maribo cheese during ripeing at $14^{\circ} \mathrm{C}$ for 6 mon

\begin{tabular}{|c|c|c|c|c|c|c|c|c|}
\hline \multirow{2}{*}{$\begin{array}{c}\text { Sensory } \\
\text { description }\end{array}$} & \multirow{2}{*}{$\begin{array}{c}\text { Treatment } \\
(\% \mathrm{w} / \mathrm{w})\end{array}$} & \multicolumn{7}{|c|}{ Ripening period (mon) } \\
\hline & & 0 & 1 & 2 & 3 & 4 & 5 & 6 \\
\hline \multirow{5}{*}{ Butyric } & Control & $00 \pm 0.00^{\mathrm{Aa}}$ & $.00 \pm 0.00^{\mathrm{Aa}}$ & $.00 \pm 0.00^{\mathrm{Aa}}$ & $4.00 \pm 0.00^{\mathrm{Ba}}$ & $4.17 \pm 0.41^{\mathrm{Aa}}$ & $4.17 \pm 0.41^{\mathrm{Aa}}$ & $.33 \pm 0.52^{\mathrm{Aa}}$ \\
\hline & $0.2 \mathrm{I}$ & $4.17 \pm 0.41^{\mathrm{Ab}}$ & $4.17 \pm 0.41^{\mathrm{Ab}}$ & $4.83 \pm 0.75^{\mathrm{Aab}}$ & $4.83 \pm 0.41^{\text {Aab }}$ & $4.83 \pm 0.75^{\mathrm{Aab}}$ & $5.00 \pm 1.10^{\mathrm{Aab}}$ & $5.17 \pm 0.75^{\mathrm{Aa}}$ \\
\hline & $0.5 \mathrm{~F}$ & $4.17 \pm 0.75^{\mathrm{Ab}}$ & $4.67 \pm 0.82^{\mathrm{Aab}}$ & $4.83 \pm 0.75^{\mathrm{Aab}}$ & $5.00 \pm 0.63^{\mathrm{Aab}}$ & $5.00 \pm 0.89^{\mathrm{Aab}}$ & $5.17 \pm 0.75^{\mathrm{Aab}}$ & $5.33 \pm 1.21^{\mathrm{Aa}}$ \\
\hline & 0.21 & $4.17 \pm 0.75^{\mathrm{Ab}}$ & $4.33 \pm 0.52^{\mathrm{Aab}}$ & $4.83 \pm 0.75^{\text {Aab }}$ & $4.67 \pm 0.82^{\mathrm{ABab}}$ & $4.83 \pm 0.41^{\mathrm{Aab}}$ & $4.83 \pm 0.98^{\mathrm{Aab}}$ & $5.17 \pm 0.75^{\mathrm{Aa}}$ \\
\hline & $0.5 \mathrm{I}$ & $4.17 \pm 0.75^{\mathrm{Ab}}$ & $4.67 \pm 0.82^{\mathrm{Aab}}$ & $4.83 \pm 0.75^{\text {Aab }}$ & $4.83 \pm 0.75^{\mathrm{Aab}}$ & $4.83 \pm 0.75^{\mathrm{Aab}}$ & $5.00 \pm 0.63^{\mathrm{Aab}}$ & $5.17 \pm 0.75^{\mathrm{Aa}}$ \\
\hline \multirow{5}{*}{ Bitterness } & $\mathrm{Col}$ & $4.00 \pm 0.00^{\mathrm{Ac}}$ & $4.50 \pm 0.55^{\mathrm{Ab}}$ & $5.00 \pm 0.41^{\mathrm{Aa}}$ & $4.50 \pm 0.55^{\mathrm{Ab}}$ & $4.33 \pm 0.52^{\mathrm{Abc}}$ & $4.00 \pm 0.41^{\mathrm{Ac}}$ & $4.00 \pm 0.41^{\mathrm{Ac}}$ \\
\hline & 0.2 PCMC & $4.00 \pm 0.00^{\mathrm{Ab}}$ & $4.83 \pm 0.98^{\mathrm{Aab}}$ & $5.33 \pm 0.82^{\mathrm{Aa}}$ & $4.67 \pm 0.82^{\mathrm{Aab}}$ & $4.67 \pm 1.21^{\mathrm{Aabc}}$ & $4.33 \pm 0.82^{\mathrm{Aab}}$ & $4.33 \pm 0.52^{\mathrm{Aab}}$ \\
\hline & 0.5 PCMC & $4.17 \pm 0.75^{\mathrm{Ac}}$ & $5.00 \pm 0.89^{\mathrm{Aabc}}$ & $5.67 \pm 1.03^{\mathrm{Aa}}$ & $5.17 \pm 0.75^{\text {Aab }}$ & $5.00 \pm 0.41^{\mathrm{Aab}}$ & $4.83 \pm 0.41^{\text {Aabc }}$ & $4.50 \pm 0.55^{\mathrm{Abc}}$ \\
\hline & $0.2 \mathrm{~N}$ & $4.00 \pm 0.63^{\mathrm{Ab}}$ & $4.33 \pm 0.52^{\mathrm{Aab}}$ & $5.17 \pm 0.75^{\mathrm{Aa}}$ & $4.67 \pm 0.82^{\text {Aab }}$ & $4.50 \pm 1.05^{\mathrm{Aab}}$ & $4.33 \pm 0.52^{\mathrm{Aab}}$ & $4.17 \pm 0.75^{\mathrm{Ab}}$ \\
\hline & $0.5 \mathrm{NCMC}$ & $4.17 \pm 0.41^{\mathrm{Ab}}$ & $4.83 \pm 0.98^{\mathrm{Aab}}$ & $5.33 \pm 0.82^{\mathrm{Aa}}$ & $5.00 \pm 0.89^{\mathrm{Aab}}$ & $4.83 \pm 0.41^{\mathrm{Aab}}$ & $4.50 \pm 1.05^{\mathrm{Aab}}$ & $4.33 \pm 0.52^{\mathrm{Aab}}$ \\
\hline \multirow{5}{*}{ Hardness } & Control & $4.50 \pm 0.55^{\mathrm{Aa}}$ & $3.83 \pm 0.41^{\mathrm{Ab}}$ & $3.83 \pm 0.41^{\mathrm{Ab}}$ & $3.50 \pm 0.84^{\mathrm{Abc}}$ & $3.33 \pm 0.52^{\mathrm{Abc}}$ & $3.17 \pm 0.41^{\mathrm{Bc}}$ & $3.00 \pm 0.41^{\mathrm{Bc}}$ \\
\hline & 0.2 PCMC & $4.50 \pm 0.55^{\mathrm{Aa}}$ & $4.17 \pm 0.98^{\mathrm{Aa}}$ & $4.00 \pm 0.89^{\mathrm{Aa}}$ & $4.17 \pm 1.17^{\mathrm{Aa}}$ & $4.00 \pm 0.63^{\mathrm{Aa}}$ & $3.83 \pm 0.41^{\mathrm{Aa}}$ & $3.67 \pm 0.52^{\mathrm{ABa}}$ \\
\hline & 0.5 PCMC & $4.83 \pm 0.41^{\mathrm{Aa}}$ & $4.67 \pm 0.82^{\mathrm{Aab}}$ & $4.33 \pm 0.52^{\mathrm{Aab}}$ & $4.00 \pm 0.63^{\mathrm{Aab}}$ & $4.17 \pm 1.17^{\mathrm{Aab}}$ & $4.00 \pm 0.41^{\mathrm{Aab}}$ & $3.83 \pm 0.41^{\mathrm{Ab}}$ \\
\hline & $0.2 \mathrm{NCMC}$ & $4.50 \pm 0.84^{\mathrm{Aa}}$ & $4.17 \pm 1.17^{\mathrm{Aa}}$ & $4.17 \pm 0.41^{\mathrm{Aa}}$ & $4.17 \pm 0.75^{\mathrm{Aa}}$ & $3.83 \pm 0.98^{\mathrm{Aa}}$ & $3.67 \pm 0.52^{\mathrm{Aa}}$ & $3.50 \pm 0.55^{\mathrm{ABa}}$ \\
\hline & $0.5 \mathrm{NCMC}$ & $4.50 \pm 0.55^{\mathrm{Aa}}$ & $4.50 \pm 0.84^{\mathrm{Aa}}$ & $4.33 \pm 0.52^{\mathrm{Aa}}$ & $4.33 \pm 0.82^{\mathrm{Aa}}$ & $4.00 \pm 0.89^{\mathrm{Aa}}$ & $3.83 \pm 0.41^{\mathrm{Aa}}$ & $3.67 \pm 0.82^{\mathrm{ABa}}$ \\
\hline \multirow{5}{*}{$\begin{array}{c}\text { Overall } \\
\text { acceptability }\end{array}$} & Control & $4.50 \pm 0.55^{\mathrm{Aa}}$ & $4.67 \pm 1.03^{\mathrm{Aa}}$ & $4.50 \pm 0.55^{\mathrm{Aa}}$ & $4.83 \pm 0.75^{\mathrm{Aa}}$ & $5.00 \pm 0.63^{\mathrm{Aa}}$ & $5.00 \pm 0.63^{\mathrm{Aa}}$ & $5.17 \pm 1.17^{\mathrm{Aa}}$ \\
\hline & 0.2 PCMC & $4.67 \pm 0.82^{\mathrm{Aa}}$ & $5.17 \pm 1.17^{\mathrm{Aa}}$ & $4.83 \pm 0.98^{\mathrm{Aa}}$ & $5.17 \pm 0.75^{\mathrm{Aa}}$ & $5.67 \pm 1.03^{\mathrm{Aa}}$ & $5.83 \pm 1.17^{\mathrm{Aa}}$ & $5.83 \pm 0.98^{\mathrm{Aa}}$ \\
\hline & 0.5 PCMC & $4.50 \pm 1.05^{\mathrm{Aa}}$ & $4.83 \pm 0.75^{\mathrm{Aa}}$ & $4.67 \pm 0.82^{\mathrm{Aa}}$ & $5.00 \pm 0.89^{\mathrm{Aa}}$ & $5.50 \pm 1.05^{\mathrm{Aa}}$ & $5.67 \pm 1.21^{\mathrm{Aa}}$ & $5.67 \pm 0.82^{\mathrm{Aa}}$ \\
\hline & $0.2 \mathrm{NCMC}$ & $4.50 \pm 1.38^{\mathrm{Ab}}$ & $5.00 \pm 0.89^{\mathrm{Aab}}$ & $5.33 \pm 0.82^{\mathrm{Aab}}$ & $5.83 \pm 0.75^{\mathrm{Aa}}$ & $6.00 \pm 0.63^{\mathrm{Aa}}$ & $6.17 \pm 0.75^{\mathrm{Aa}}$ & $6.17 \pm 0.75^{\mathrm{Aa}}$ \\
\hline & $0.5 \mathrm{NCMC}$ & $4.67 \pm 1.21^{\mathrm{Ab}}$ & $4.83 \pm 0.75^{\mathrm{Aab}}$ & $5.17 \pm 0.75^{\mathrm{Aab}}$ & $5.83 \pm 0.75^{\mathrm{Aab}}$ & $5.83 \pm 1.17^{\mathrm{Aab}}$ & $6.00 \pm 1.10^{\mathrm{Aa}}$ & $6.00 \pm 0.89^{\mathrm{Aa}}$ \\
\hline
\end{tabular}

Means with different superscripts in a row (a-c) and column (A-B) are significant at $p<0.05$.

Control $=$ Maribo cheese.

PC: powdered chitosan-added Maribo cheese.

NPC: nanopowdered chitosan-added Maribo cheese. 


\section{Sensory attribute}

Sensory attributes of NCMC during ripening at $14^{\circ} \mathrm{C}$ for 6 mon are shown in Table 5. In butyric off-flavor, $0.2 \%$ NCMC and control were increased from 4.17 to 5.17 and from 4.00 to 4.33 during 6 mon ripening, respectively. Bitterness increased until 2 mon, thereafter it was decreased in all samples. In the process of protein degraded to amino acid, dipeptide could cause bitterness of cheese. In addition, cheeses added-chitosan powder had a slightly bitter taste than control because of the original bitter taste of nanopowdered and powdered chitosans. Hardness of control was rapidly decreased at 1 mon. Chitosan-added cheeses were significantly harder than control at 5 and 6 mon $(p<0.05)$. Hardness was 4.50 at initial and after ripening it was decreased to 3.50 in $0.2 \%$ NCMC and 3.00 in control. Hardness of chitosan-added samples was increased with increasing chitosan concentration. Overall acceptability was also affected by addition of nanopowdered and powdered chitosans in cheese samples, but there were not significantly different among the samples. After the ripening fore 2 mon, $0.2 \% \mathrm{NCMC}$ had the best overall acceptability among the samples (6.17). Altieri et al. (2005) reported that sensory analysis between $0.075 \%$ chitosanadded Mozzarella cheese and chitosan-free cheeses were not different. According to Seo et al. (2011), 1-9\% nanochitosan-added milks did not significantly influenced bitter taste and overall scores $(p>0.05)$.

\section{Conclusion}

The current study was designed to develop on NPCadded Maribo cheeses and to evaluate the effect of adding NPC in the chemical, texture, and sensory properties during ripening. The data on the SCFA, color, texture, and sensory analysis obtained from the current study indicated that $0.2 \%$ concentration of NPC could be applicable in NPC-added functional cheese development. Therefore, the present study may provide the possibility of NPCadded Maribo cheese manufacture.

\section{Acknowledgments}

The present study was supported by a grant from Korea Institute of Planning and Evaluation for Technology in Food, Agriculture, Forestry and Fisheries in GyeonggiDo, Republic of Korea (111020-03-3-HD120).
1. Cichoscki, A. J., Eunice, V., Alice, T. V., Marýìa, E. T., and José, M. F. (2002) Characterization of Prato cheese, a Brazilian semi-hard cow variety: Evolution of physico-chemical parameters and mineral composition during ripening. Food Control. 13, 329-336.

2. Altieri, C., Scrocco, C., Sinigaglia, M., and Del Nobile, M. A. (2005) Use of chitosan to prolong mozzarella cheese shelf life. J. Dairy Sci. 88, 2683-2688.

3. AOAC. (2000) Official methods of analysis. 19th ed. Association of Official Analytical Chemists, Washington, DC, USA.

4. Brito, C., Astete, M. A., Pinto, M., and Molina, L. H. (2000) Maribo cheese manufactured with concentrated milk: Characteristics, maturation and yield. Int. J. Dairy Technol. 53, 612.

5. Chaudhry, Q., Scotter, M., Blackburni, J., Ross, B., Boxall, A., Castle, L., Aitken, R., and Watkins, R. (2008) Applications and implications of nanotechnologies for the food sector. Food Addit. Contam. 25, 241-258.

6. Fox, P. F. (1989) Proteolysis during cheese manufacture and ripening. J. Dairy Sci. 72, 1379-1400.

7. Gallaher, C. M., Munion, J., Hesslink, R. Jr., Wise, J., and Gallaher, D. D. (2000) Cholesterol reduction by glucomannan and chitosan is mediated by changes in cholesterol absorption bile acid fat excretion in rats. J. Nutr. 130, 2753-2759.

8. Hayashi, K. and Ito, M. (2001) Antidiabetic action of low molecular weight chitosan in genetically obese diabetic KK-Ay mice. Biol. Pharm. Bull. 25, 188-192.

9. Kwak, H. S., Jeon, I. J., and Park, D. (1990) Effect of food grade porcine pancreatic lipase on the production of shortchain fatty acids and its contribution. Kor. J. Food Sci. Technol. 22, 248-254.

10. Lin, J. C. C. and Jeon, I. J. (1987) Effects of commercial food grade enzymes on free fatty acid profiles in granular cheddar cheese. J. Food. Sci. 52, 79-83.

11. McSweeney, P. L. H. (2004) Biochemistry of cheese ripening. Int. J. Dairy Technol. 57, 127-144.

12. Miura, T., Usami, M., Tsuura, Y., Ishida, H., and Seino, Y., (1995) Hypoglycemic and hypolipidemic effect of chitosan in normal and neonatal streptozotocin-induced diabetic mice. Biol. Pharm. Bull. 18, 1623-1625.

13. Park, H. S., Jeon, B. J., Ahn, J., and Kwak, H. S. (2007) Effects of nanocalcium supplemented milk on bone metabolism in ovariectomized rats. Asian-Aust. J. Animal. Sci. 20, 12661271.

14. Park J. H., Hong, E. K., Ahn, J., and Kwak, H. S. (2010) Properties of nanopowdered chitosan and its cholesterol lowering effects in rats. Food Sci. Biotechnol. 19, 1457-1462.

15. Qin, C., Du, Y., Xiao, L., Li, Z., and Gao, X. (2002) Enzymic preparation of water-soluble chitosan and their antitumor activity. Int. J. Biol. Macromol. 31, 111-117.

16. Rohm, H. and Jaros, D. (1996) Colour of hard cheese. Z Lebensm Unters Fosch. 203, 241-244.

17. Robinson, R. K. (1995) A color guide to cheese and fermented milk products. Chapman \& Hall. London. U.K. 
18. Seo, M. H., Chang, Y. H., Lee, S., and Kwak, H. S. (2011) The physicochemical and sensory properties of milk supplemented with ascorbic acid-soluble nano-chitosan during storage. Int. J. Dairy Technol. 64, 57-63.

19. Seo, M. H., Park, J. H., and Kwak, H. S. (2010) Antidiabetic activity of nanopowdered chitosan in db/db Mice. Food Sci. Biotechnol. 19, 1245-1250.

20. Seo M. H., Lee, S. Y., Chang, Y. H., and Kwak, H. S. (2009) Physicochemical, microbial, and sensory properties of yogurt supplemented with nanopowdered chitosan during storage. $J$. Dairy Sci. 92, 5907-5916.
21. Sugimoto, M., Morimoto, M., Sashiwa, H., Saimoto, H., and Shigemasa, Y. (1998) Preparation and characterization of water-soluble chitin and chitosan derivatives. Carbohyd. Polym. 36, 49-59.

22. Tunick, M. H. and Van Hekken, D. L. (2002) Torsion gelometry of cheese. J. Dairy Sci. 85, 2743-2749.

23. Zhang, W., Zhang, J., Jiang, Q., and Xia, W. (2013) The hypolipidemic activity of chitosan nanopowder prepared by ultrafine milling. Carbohyd. Polym. 95, 487-491. 